Supplement of The Cryosphere, 16, 1-15, 2022

https://doi.org/10.5194/tc-16-1-2022-supplement

(C) Author(s) 2022. CC BY 4.0 License.

(c) (i)

Supplement of

\title{
Assessing volumetric change distributions and scaling relations of retro- gressive thaw slumps across the Arctic
}

Philipp Bernhard et al.

Correspondence to: Philipp Bernhard (bernhard@ifu.baug.ethz.ch)

The copyright of individual parts of the supplement might differ from the article licence. 


\section{Propbility density function of area and volumetric change rates}

To compute the rollover, cutoff, exponetial decay coefficients we fitted a three-parameter inverse Gamma function defined by:

$p d f\left(X_{R T S} \mid \rho, a, s\right)=\frac{1}{a \Gamma(\rho)}\left(\frac{1}{X_{R T S}-s}\right)^{\rho+1} \exp \left(-\frac{a}{X_{R T S}-s}\right)$

where $X_{R T S}$ is either the yearly area or volume change and $\Gamma(\rho)$ is the gamma function of $\rho$. The determined values of the

5 fitting parameters as well as the rollover, cutoff and exponential decay coefficients can be seen in Table S1 and S2.

Table S 1. Rollover Cutoff and Exponential decay for yearly area change rate.

\begin{tabular}{|c|c|c|c|c|c|c|}
\hline Area & $\begin{array}{l}\text { Rollover } \\
{\left[10^{2} \mathrm{~m}^{2} \mathrm{yr}^{-1}\right]}\end{array}$ & $\begin{array}{l}\text { Cutoff } \\
{\left[10^{3} \mathrm{~m}^{2} \mathrm{yr}^{-1}\right]}\end{array}$ & Exp.decay & $\rho$ & $\begin{array}{l}\mathrm{a} \\
{\left[10^{3}\right]}\end{array}$ & $\begin{array}{l}\mathrm{s} \\
{\left[10^{3}\right]}\end{array}$ \\
\hline Peel & $4.96 \pm 0.48$ & $1.98 \pm 0.94$ & $2.4 \pm 0.4$ & 1.45 & 1.47 & -0.11 \\
\hline $\mathrm{Bar}$ & 66 & 3.95 & 3.0 & 1.76 & 3.60 & -0.21 \\
\hline Elle & $46+098$ & $41 \pm 2.74$ & $3.2=$ & 1.49 & 3.10 & -0.10 \\
\hline & & & & 1.82 & 0.76 & 0.03 \\
\hline & & & & 12.9 & 27.62 & 1.38 \\
\hline Che & 50 & 15 & 3.2 & 29.59 & 100.76 & -2.82 \\
\hline Таy & 4.02 & $1.15=$ & $3.0 \pm$ & 0.85 & 0.34 & -0.19 \\
\hline Yamal/Gydan & $3.92 \pm 0.73$ & $1.36 \pm 0.20$ & $3.7 \pm 1.2$ & 4.26 & 4.34 & -0.41 \\
\hline
\end{tabular}

Table S 2. Rollover Cutoff and Exponential decay for yearly volumetric change rate.

\begin{tabular}{l|l|l|l|l|l|l|} 
Area & $\begin{array}{l}\text { Rollover } \\
{\left[10^{2} \mathrm{~m}^{3} \mathrm{yr}^{-1}\right]}\end{array}$ & $\begin{array}{l}\text { Cutoff } \\
{\left[10^{3} \mathrm{~m}^{3} \mathrm{yr}^{-1}\right]}\end{array}$ & Exp.decay & $\rho$ & $\mathrm{a}$ & $\mathrm{s}$ \\
{$\left[10^{3}\right]$} & {$\left[10^{3}\right]$} \\
\hline Peel & $15.21 \pm 1.86$ & $3.31 \pm 1.60$ & $1.9 \pm 0.1$ & 0.97 & 3.71 & -0.37 \\
Banks & $16.57 \pm 1.14$ & $5.34 \pm 2.96$ & $2.0 \pm 0.2$ & 0.95 & 3.33 & -0.05 \\
Ellesmere & $29.83 \pm 4.34$ & $3.97 \pm 2.73$ & $1.9 \pm 0.2$ & 1.28 & 7.17 & -0.32 \\
Tuktuyaktuk & $7.28 \pm 0.67$ & $1.35 \pm 0.84$ & $2.3 \pm 0.2$ & 2.15 & 2.89 & -0.20 \\
Noatak & $5.83 \pm 3.36$ & $1.58 \pm 0.45$ & $2.1 \pm 0.3$ & 0.73 & 0.63 & -0.14 \\
Chukotka & $10.42 \pm 4.70$ & $2.19 \pm 0.90$ & $2.6 \pm 0.5$ & 7.22 & 32.29 & -2.55 \\
Taymyr & $9.63 \pm 1.71$ & $2.06 \pm 1.40$ & $2.3 \pm 0.5$ & 0.97 & 1.32 & 0.26 \\
Yamal/Gydan & $10.23 \pm 2.93$ & $2.41 \pm 0.88$ & $2.8 \pm 0.6$ & 4.25 & 12.54 & -1.23
\end{tabular}




\section{Additional Figures}

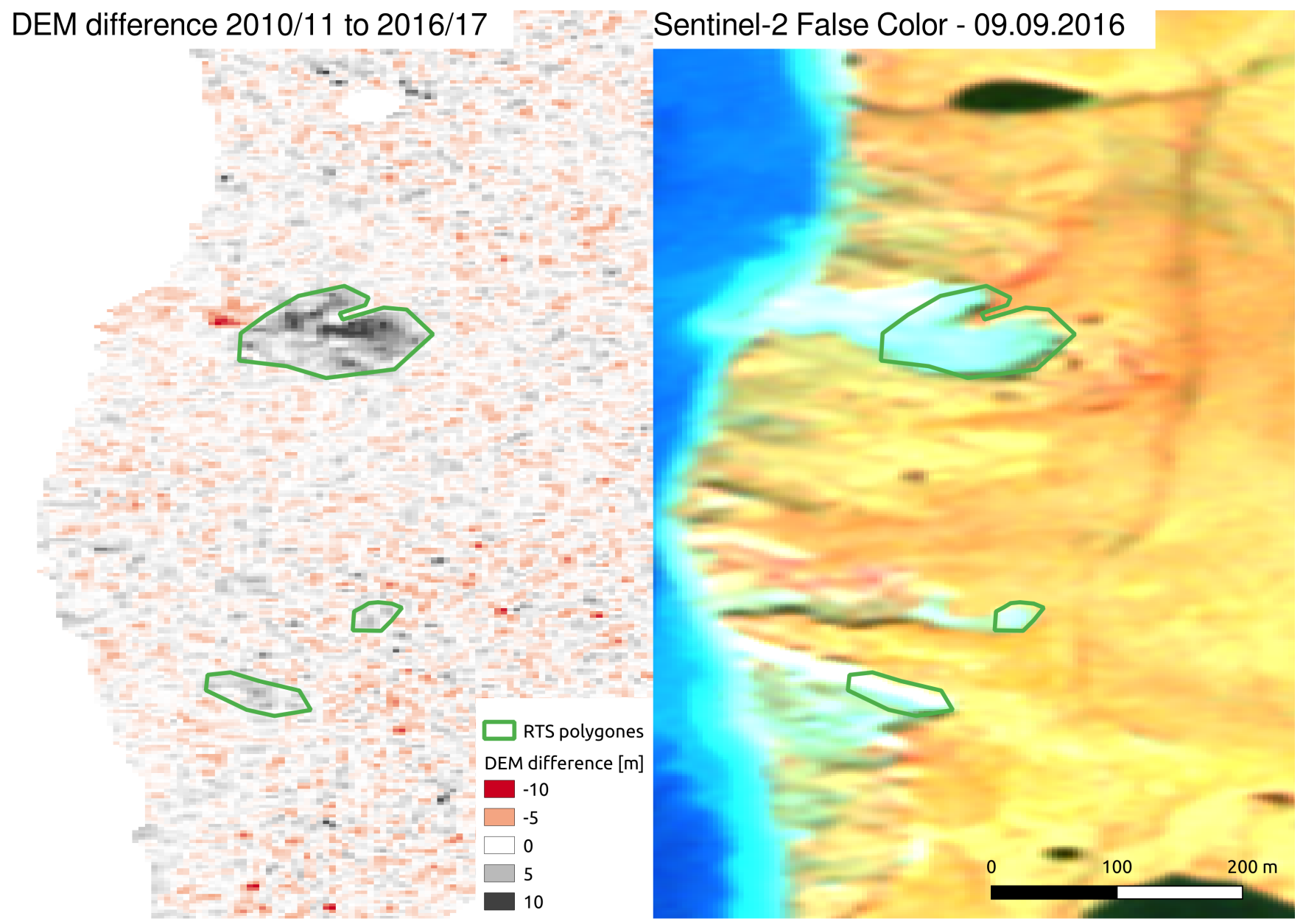

Figure S 1. Example of RTS polygones in the Chukotka study region located at N65.93 W-178.82. Left: DEM difference image between winter 2010/11 and 2016/17. Right: False color Sentinel-2 image taken on 09.09.2016. 


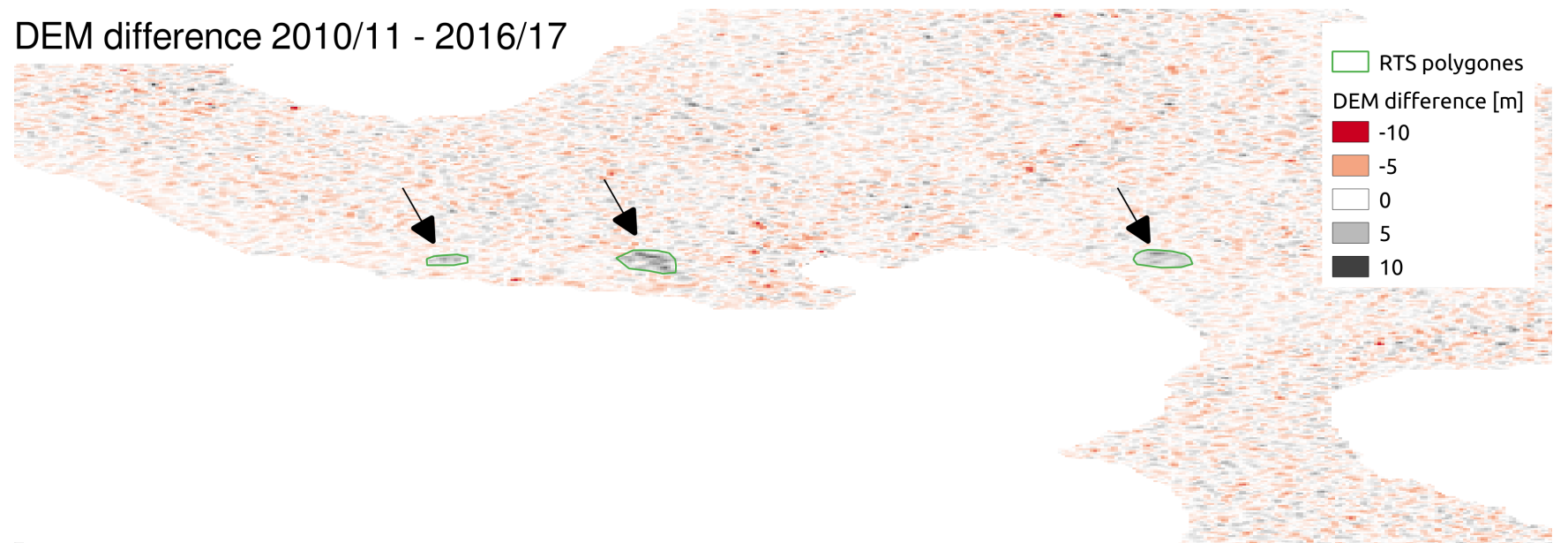

\section{Sentinel-2 False Color - 06.08.2016}

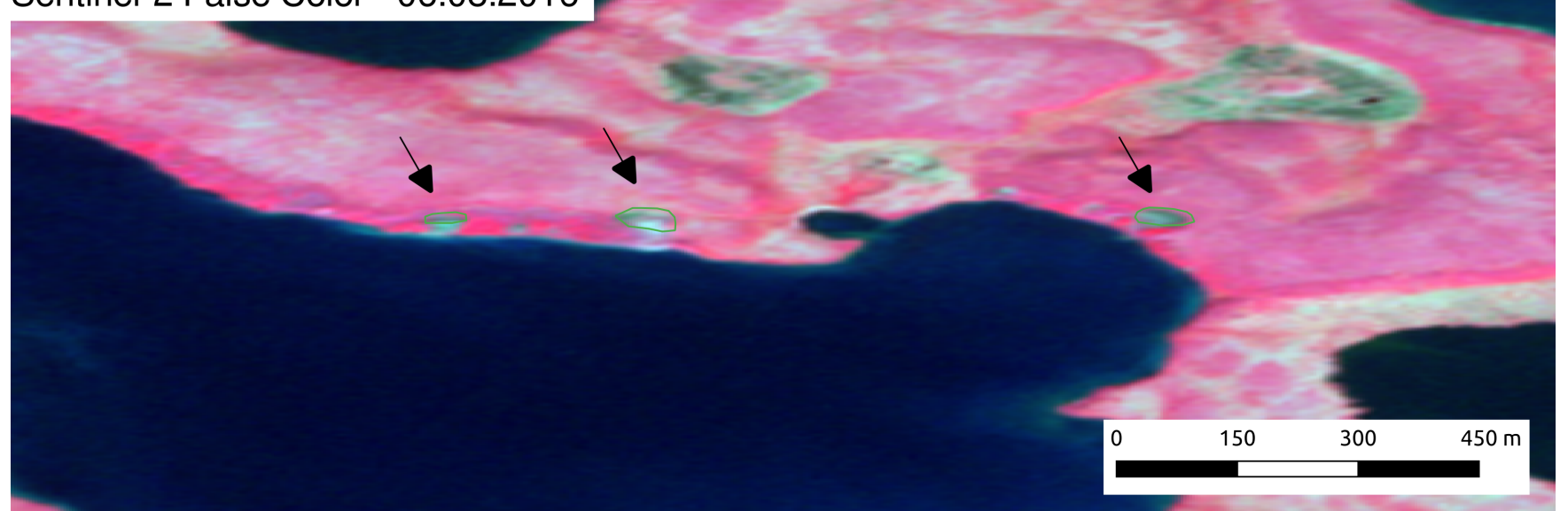

Figure S 2. Example of RTS polygones in the Tuktoyaktuk study region located at N69.08 W-134.02. The black arrows indicate the RTS lcoations Left: DEM difference image between winter 2010/11 and 2016/17. Right: False color Sentinel-2 image taken on 06.08.2016. 


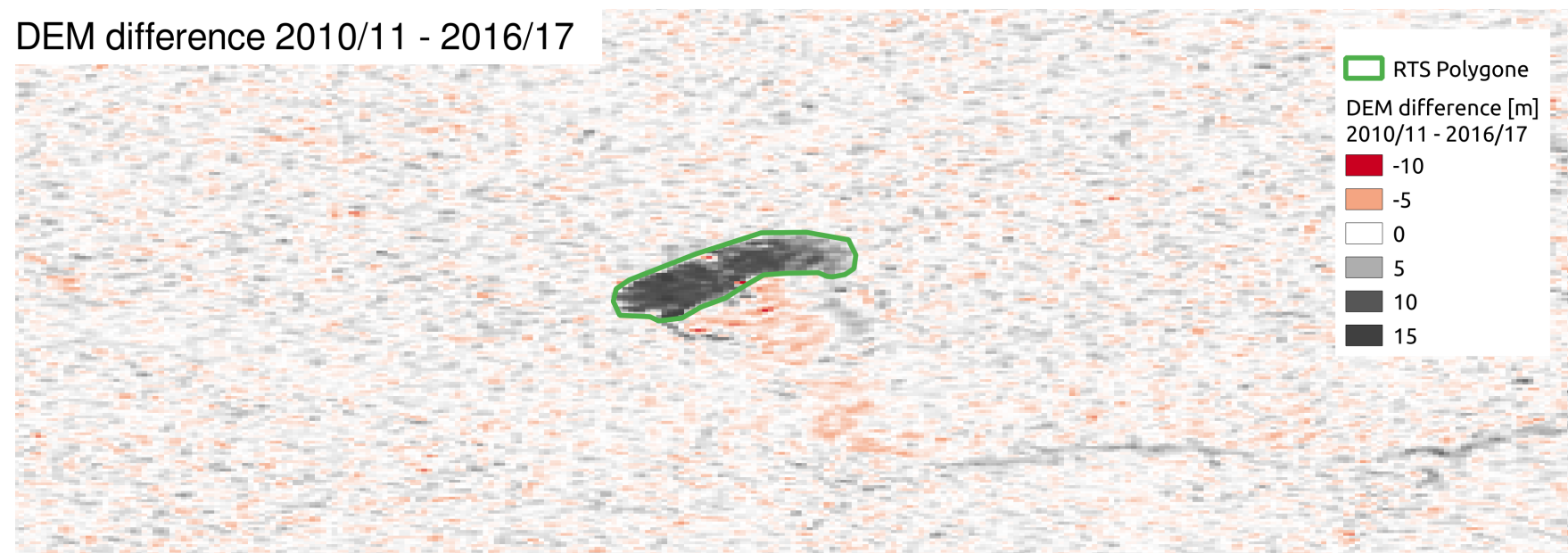

\section{Sentinel-2 False Color - 06.08.2016}

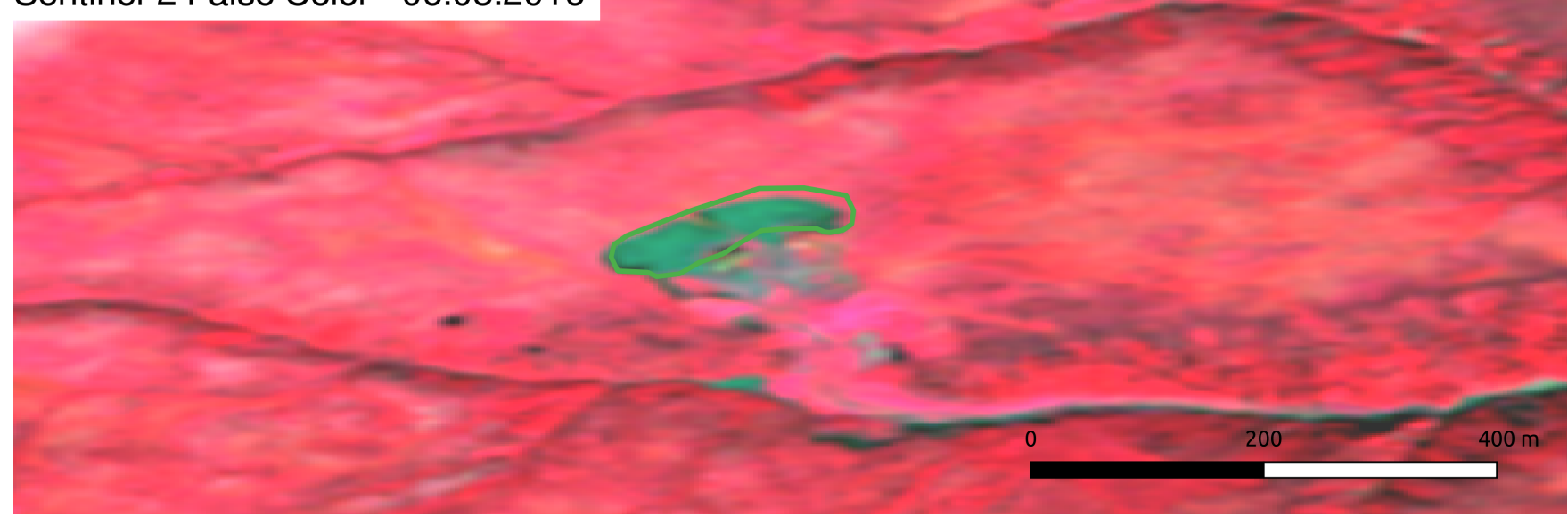

Figure S 3. Example of RTS polygones in the Peel study region located at N67.26 W-135.27. Left: DEM difference image between winter 2010/11 and 2016/17. Right: False color Sentinel-2 image taken on 06.08.2016. 


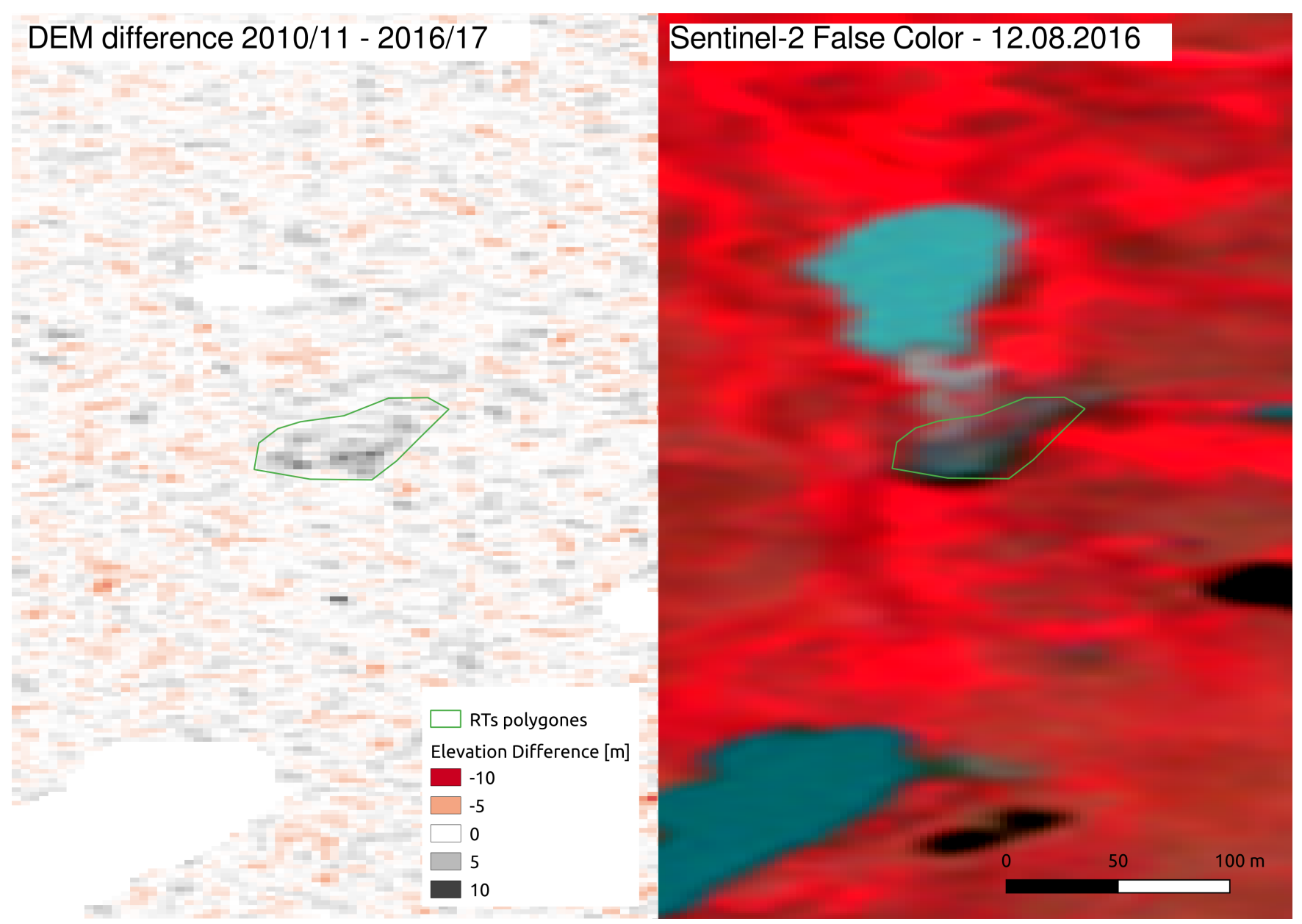

Figure S 4. Example of RTS polygones in the Yamal study region located at N71.09 W70.40 . Left: DEM difference image between winter 2010/11 and 2016/17. Right: Fasle color Sentinel-2 image taken on 12.08.2016. 


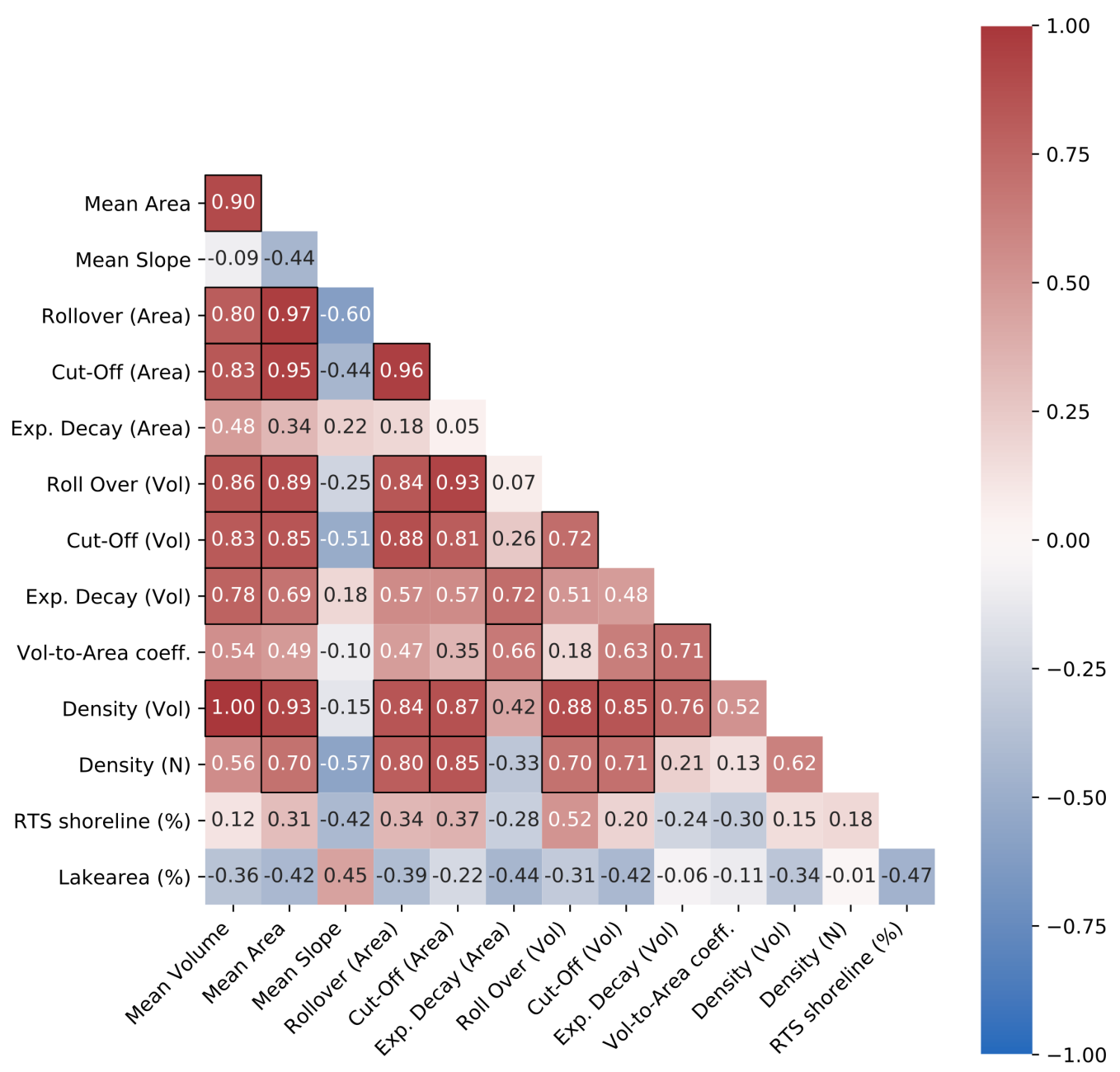

Figure S 5. Correlation coefficients between all computed quantities of all areas. Values below -0.64 and above 0.64 are statistically significant (t-Test with a p-value $<0.05)$. 
(a)
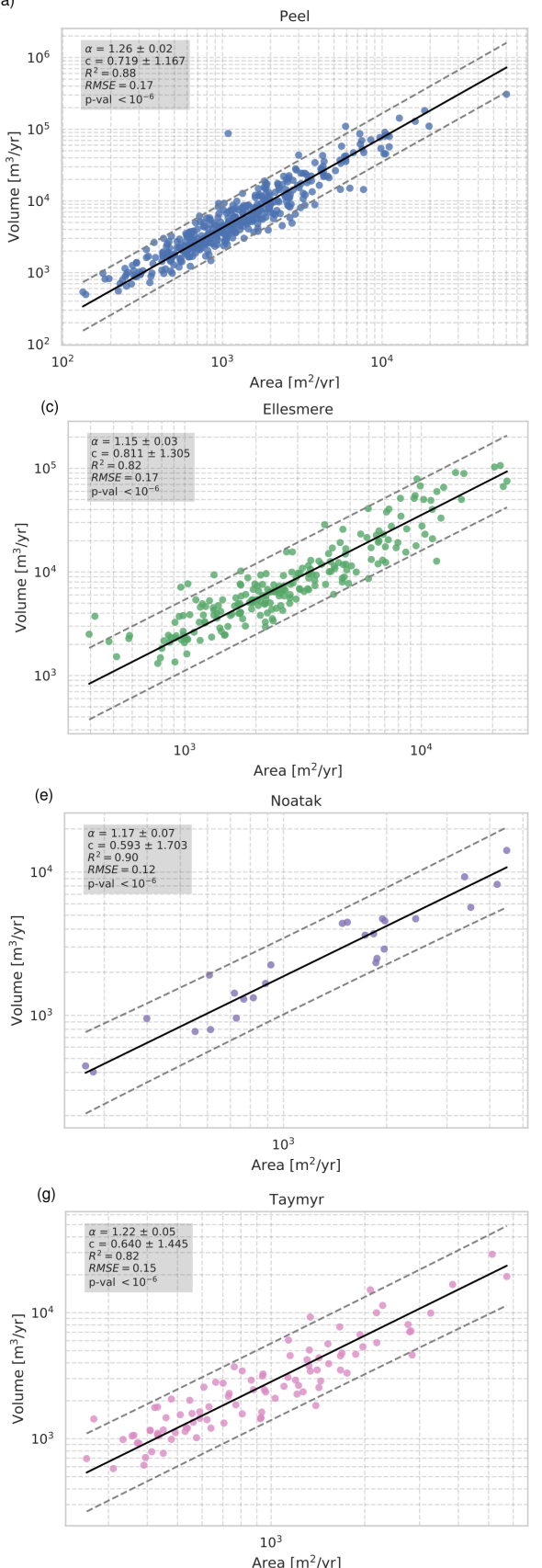

(b)
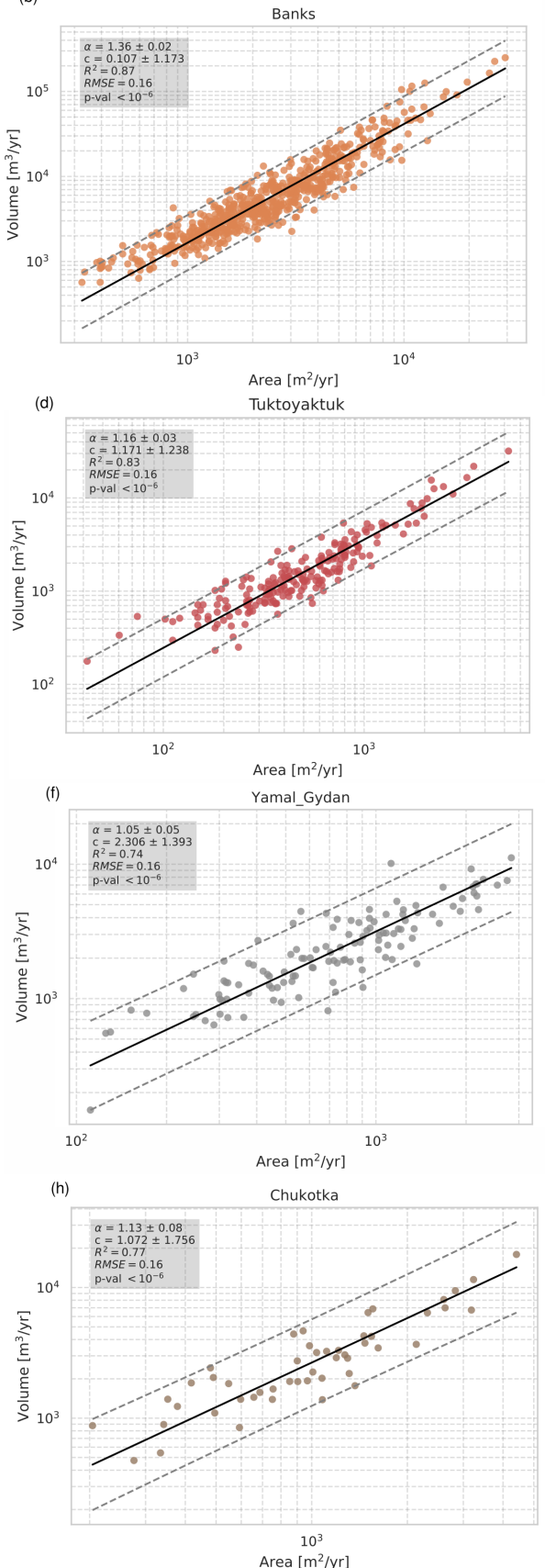

Figure S 6. Area to volume scaling for each study area. 
(a)

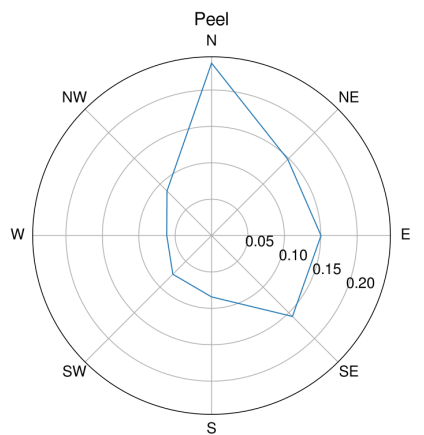

(c)
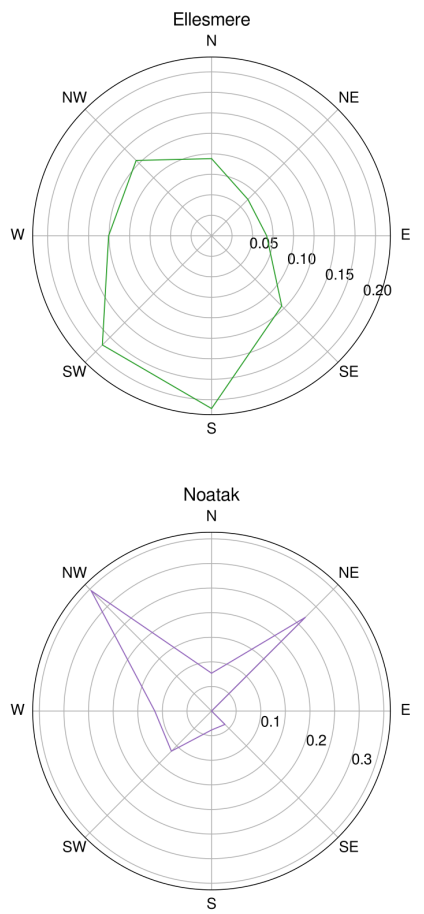

(g)

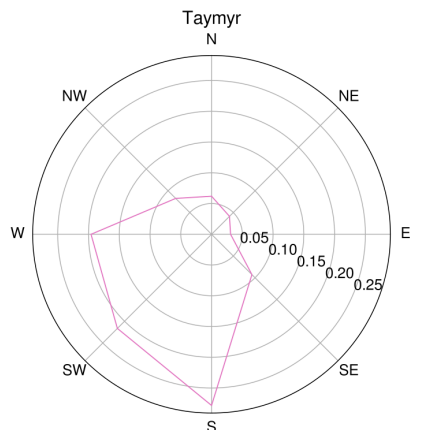

(b)

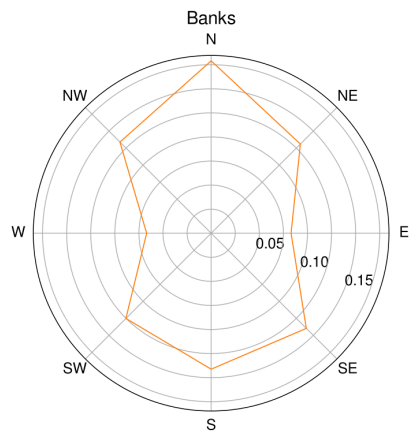

(d)

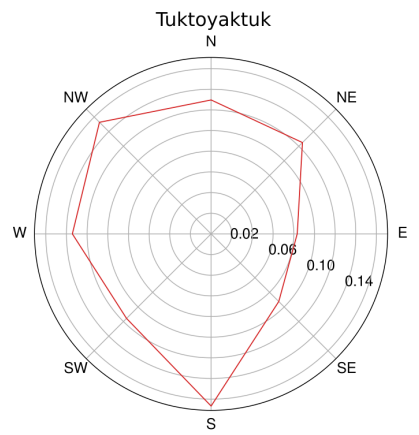

(f)

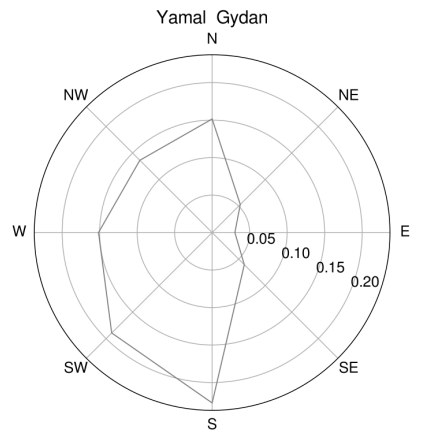

(h)

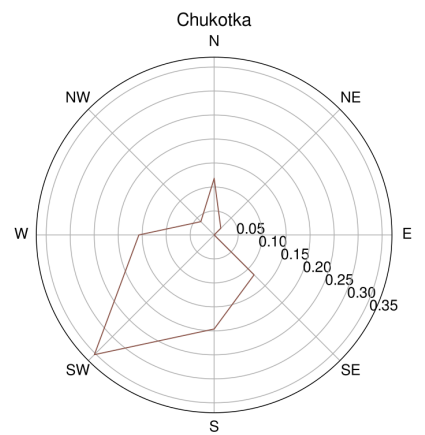

Figure S 7. Aspect distribution of all study areas. 